\title{
Über einige neue Carbazolderivate
}

von

\section{Bruno Levy}

(Vorgelegt in der Sitzung am 11. Jänner 1912.)

Graebe und Behaghel ${ }^{1}$ haben Methyl und Äthylcarbazol dargestellt, indem sie Carbazolkalium mit Jodalkyl auf 170 bis $190^{\circ}$ im Bombenrohr erhitzten; Ehrenreich hat kürzlich im hiesigen Laboratorium die Beobachtung gemacht, daß Carbazolkalium schon bei Zimmertemperatur mit Methyljodid reagiert und nahezu quantitativ bei dieser Temperatur in Methylcarbazol übergeführt wird. Es war zu erwarten, daß auch andere Halogenalkyle auf Carbazolkalium schon bei niedrigerer Temperatur als der von Graebe und Behaghel gewählten einwirken würden. Herr Prof. Goldschmiedt hat mich daher veranlaßt, Untersuchungen in dieser Richtung anzustellen.

Zur Untersuchung gelangten folgende Halogenalkyle: Äthyljodid, norm. Propyljodid, Isopropyljodid, norm. Butyljodid, sec. Butyljodid, Isobutyljodid, Isoamyljodid, sec. Amyljodid, Allyljodid, Benzylchlorid, Triphenylmethylchlorid. Es zeigte sich, daß die Reaktionsgeschwindigkeit, wie zu erwarten war, von der Natur des Alkyls in hohem Maße abhängig ist; mit steigendem Molekulargewicht nimmt dieselbe rasch $a b$, wird aber durch doppelte Bindung beträchtlich erhöht; auch Benzylchlorid ist sehr reaktionsfähig, Verzweigung der Kohlenstoffkette im Alkyl bewirkt Depression der Reaktionsgeschwindigkeit. Bemerkenswert ist, daß auch Triphenylmethylchlorid, bei dem man aus sterischen Gründen eine Einwirkung auf

1 Annalen, 202, $23(1880)$. 
Carbazoikalium nicht erwaten sollte, doch schon bei der Temperatur des siedenden Benzols unter Bildung ron Triphenymethylcarbazol reagiert. Äthylcarbazol wurde mit guter Ausbeute durch Einwirkung von Äthyljodid auf Carbazolkalium während 24 Stunden bei Zimmertemperatur gewonnen. Eine Erwärmung der Reaktionsmasse, wie sie bei der Bildung des Methylcarbazols auftritt, wurde beim Zufügen des Carbazolkaliums und Äthyljodides nicht beobachtet.

Propyl und Isopropylcarbazol bildeten sich innerhalb drei Tagen bei Zimmertemperatur nur in geringen Mengen, bei fünfstündigem Erwärmen des Carbazolkaliums mit dem betreffenden Alkyliodid erhielt ich eine gute Ausbeute an Propyl- und Isopropylcarbazol, dabei erwies sich das Propyljodid, wenn auch in geringem Maße, reaktionsfïhiger als das Isopropyljodid. Die Butyljodide reagierten innerhalb 3 Tagen bei Zimmertemperatur fast gar nicht, auch nach fünfstündigem Erwärmen war die Umsetzung noch recht unvollkommen, am besten reagierte das normale Butyljodid, am schlechtesten das tertiäre. Bei dem sec. Amyl- und Isoamyljodid ließen sich bei Zimmertemperatur nach 3 Tagen nur Spuren eines Reaktionsproduktes beobachten; nach fünfstündigem Erwärmen auf dem Wasserbad war die Ausbeute klein. Die Reaktionsfähigkeit der heiden Halogenalkyle war fast gleich. Allyljodid reagierte mit Carbazolkalium schon bei Zimmertemperatur, wobei beträchtliche Erwärmung eintrat. Benzylchlorid gab ebenfalls schon bei Zimmertemperatur Benzylcarbazol; auf dem Wasserbad erwärmt war die Reaktion nach 5 Stunden fast vollständig.

\section{Experimenteller Teil.}

\section{Äthylcarbazol.}

Überschüssiges Jodäthyl wurde unter zeitweisem Umrühren auf $5 g$ fein verteiltes Carbazolkalium bei Zimmertemperatur einwirken gelassen. Nach Verlauf von 24 Stunden wurde die Lösung vom Niederschlag getrennt, das überschüssige Jodäthyl abdestilliert, wobei geringe Mengen Äthylcarbazols erbalten wurden, die Hauptmenge des Reaktionsproduktes befand sich im Niederschlag und wurde daraus durch Extraktion 
mit Alkohol gewonnen. Nach dem Umkrystallisieren zeigte der Körper den von Graebe und Behaghel für Äthylcarbazol angegebenen Schmelzpunkt von $68^{\circ}$.

\section{Propylcarbazol.}

$\bar{g} g$ fein gepulvertes Carbazolkalium wurden 3 Tage auf einen Uberschuß von norm. Propyljodid bei Zimmertemperatur einwirken gelassen, hierauf der Niederschlag von der Lösung getrennt; nach Abdestillieren des überschüssigen Propyljodids erhielt ich $0.6 g$ Rückstand, der größtenteils aus Propylcarbazol bestand. Der im Propyljodid ungelöst gebliebene Teil bestand aus unverändertem Carbazolkalium.

5. Carbazolkalium wurden mit überschüssigem Propy jodid 5 Stunden auf dem Wasserbad erwärmt und darauf die Reaktion unterbrochen. Die Hauptmenge des Propylcarbazols befand sich im überschüssigen Propyljodid gelöst und wurde nach wiederholtem Umkrystallisieren aus Athylalkohol in farblosen Nadeln vom Schmelzpunkt $50^{\circ}$ erhalten. Aus dem im Propyljodid ungelösten Teil wurden noch geringe Mengen von Propylcarbazol gewonnen. Ausbeute in Summe $4 g$. Durch Lösen von Propylcarbazol in wenig Alkohol und Hinzufugen einer alkoholischen Lösung von Pikrinsäure wurde ein in schönen, dunkelroten Nadeln krystallisierendes Pikrat gewonnen. Nach einmaligem Umkrystallisieren war dasselbe rein und schmolz bei $98^{\circ}$.

$0 \cdot 2336 \mathrm{~g}$ der über Schwefeisäure getrockncten Substanz gaben $1+4 \mathrm{~cm}^{3}$ Stickstoff bei $28^{\circ}$ und $7 \pm 2 m m$ Druck.

In 100 Teilen

$$
\text { N } \ldots \ldots \ldots \ldots \underbrace{\text { Gefunden }}_{6 \cdot 7 B} \quad \underbrace{\begin{array}{c}
\text { Berechnet für } \\
\mathrm{C}_{5,5} \mathrm{H}_{15} \mathrm{~N}
\end{array}}_{6 \cdot 7}
$$

\section{Isopropylcarbazol.}

5 g Carbazolkalium wurden 3 Tage unter zeitweisem Umrühren auf überschüssiges Isopropyljodid bei Zimmertemperatur einwirken gelassen, wobei der größte Teil von 
Carbazolkalium unverändert blieb, es konnte nur $0.4 \mathrm{~g}$ Isopropylcarbazol gewonnen werden.

ò $g$ Carbazolkalium wurden mit überschüssigem Isopropyljodid 5 Stunden auf dem Wasserbad erwärmt; beim Aufarbeiten der Reaktionsmasse wurden $3 \xi$ Isopropylcarbazol gewonnen. Schmelzpunkt $120^{\circ}$. Auch hier war die Hauptmenge des gebildeten Isopropylcarbazols im überschüssigen Isopropyljodid gelöst. Das Pikrat wurde auf die gleiche Weise wie beim vorhergehenden Präparat, erhalten; es krystallisiert in dunkelroten Nadeln vom Schmelzpunkt $143^{\circ}$.

$11-2292$ o der uiber Schwefelsäure getrockneten Substanz gahen $14.1 \mathrm{~cm}^{3}$ Stickstoff bei $24^{\circ}$ und $7+4 m m$ Druck.

In 100 Teilen:

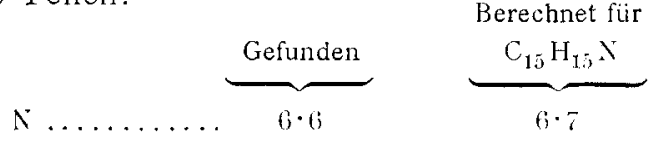

\section{Normales Butylcarbazol.}

$5 g$ Carbazolkalium wurden bei Zimmertemperatur mit norm. Butyljodid 3 Tage stehen gelassen, die Ausbeute an Butylcarbazol betrug $0.4 \mathrm{~g} .5 \mathrm{~g}$ Carbazolkalium wurden mit einem Überschuß an norm. Butyljodid 5 Stunden auf dem Wasserbad erwärmt; auf die gewöhnliche Weise aufgearbeitet, erwies sich der im Butyljodid ungelöst gebliebene Rückstand als Jodkalium und Carbazolkalium, während das gebildete Butylcarbazol sich im überschüssigen Butyljodid völlig gelöst befand. Das Butylcarbazol wurde nach häufigem Umkrystallisieren aus Alkohol in langen nahezu farblosen Nadeln rom Schmelzpunkt $58^{\circ}$ erhalten. Ausbeute $2 g$. Das Pikrat krystallisiert in langen, rubinroten Nadeln vom Schmelzpunkt $89^{\circ}$.

$0-2137 \mathrm{~g}$ der ïber Schwefelsäure getrockneten Substanz gaben $12 \cdot 8 \mathrm{~cm}$ "Stickstoff bei $23^{\circ}$ und 140 mm Druck.

In 100 Teilen:

Berechnet für

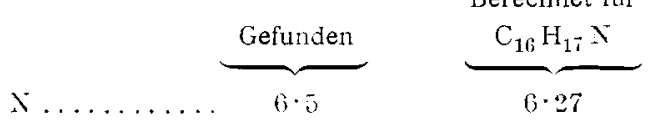




\section{Sec. Butylcarbazol.}

Die Ausbeute an Butylcarbazol bei fünftägigem Einwirken von sec. Butyljodid auf $5 g$ Carbazolkalium betrug $0 \cdot 3 g$. $5 g$ Carbazolkalium gaben nach fünfstündigem Erwärmen mit überschüssigem sec. Butyljodid auf dem Wasserbade zirka $2 g$ sec. Butylcarbazol, welches als Öl erhalten wurde und nicht zum Krystallisieren gebracht werden konnte. Das Pikrat krystallisiert aus Alkohol in kurzen, roten Nadeln vom Schmelzpunkte $64^{\circ}$. Es wurde versucht durch Zersetzen des Piklates mittels Ammoniak das sec. Butylcarbazol in krystallisiertem Zustande zu erhalten, was jedoch ebenfalls nicht gelang.

1) $1313 \mathrm{~g}$ des über Schwefelsäure getrockneten Pikrates gaben $15 \cdot 1 \mathrm{~cm}^{3}$ Stickstoff bei $23^{\circ}$ und $746 \mathrm{~mm}$ Druck.

In 100 Teilen:

$$
\mathrm{N} \ldots \ldots \ldots \ldots \underbrace{\text { Gefunden }}_{12 \cdot 6} \quad \underbrace{{ }_{1 \mathrm{if}} \mathrm{H}_{17} \mathrm{~N} . \mathrm{C}_{6} \mathrm{H}_{3}\left(\mathrm{NO}_{2}\right)_{3} \mathrm{O}}_{12 \cdot 39}
$$

\section{Isobutylcarbazol.}

Bei der Einwirkung von Carbazolkalium auf Isobutyljodid in der Kälte unter Einhaltung der allgemein gewählten Bedingungen wurden $0.2 \mathrm{~g}$ Isobutylcarbazol gewonnen. Bei fünfstündigem Erhitzen auf dem Wasserbade wurden $1.5 \mathrm{~g}$ Isobutylcarbazol als Öl erhalten, das ebenfalls nicht zum Krystallisieren zu bringen war. Das Pikrat krystallisiert in hellroten Nadeln vom Schmelzpunkt $177^{\circ}$.

$0 \cdot 1425$ g des über Schwefelsäure getrockneten Pikrates gaben $17 \cdot 4 \mathrm{~cm}$ Stickstoff bei $23^{\circ}$ und $744 m m$ Druck.

In 100 Teilen:

$$
\mathrm{N} \ldots \ldots \ldots \ldots \underbrace{\text { Gefunden }}_{12 \cdot \tau} \quad \underbrace{\mathrm{C}_{16} \mathrm{H}_{17} \mathrm{~N} \cdot \mathrm{C}_{6} \mathrm{H}_{3}\left(\mathrm{NO}_{2}\right)_{3} \mathrm{O}}_{12 \cdot 39}
$$

\section{Isoamylcarbazol.}

Bei der Einwirkung von Isoamyljodid auf Carbazolkalium wurden nur Spuren eines Reaktionsproduktes erbalten. In der 
Wärme gaben is Carbazolkalium in 5 Stunden $1 \cdot 3 g$ Isoamylcarbazol in Form eines Öles. Das Pikrat krystallisiert in dunkelroten Nadeln vom Schmelzpunkt $85^{\circ}$.

$1 \cdot 13,52$.5 des über Schwefolsäure getrockncten Pikrates gaben $15 \cdot 3 \mathrm{~cm}^{3}$ Sticlssitrff bei $23^{\circ}$ und $751 \mathrm{~mm}$ Druck.

In 100 'Teilen:

Berechnet für

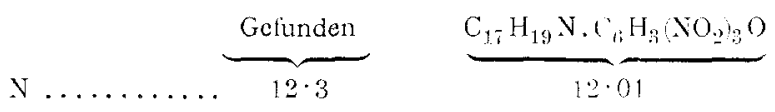

\section{Sec. Amylcarbazol.}

Sec. Amyljodid gab bei der Einwirkung auf Carbazolkalium bei Zimmertemperatur nur Spuren eines Reaktionsproduktes. Bei Wasserbadtemperatur wurden bei den gewöhnlichen Bedingungen aus $5 g$ Carbazolkalium $1 \cdot 2 \mathrm{~g}$ sec. Amylcarbazol als Öl erhalten. Das Pikrat krystallisiert in roten Nadeln rom Schmelzpunkt $93^{\circ}$.

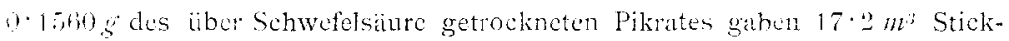
stoff bei $23^{\circ}$ und $7+6$ mm Druck.

In 1100 Teilen:

Berechnet firir

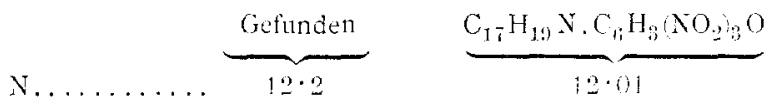

\section{Allylcarbazol.}

Allyljodid wirkt auf Carbazolkalium schon bei Zimmertemperatur lebhaft ein, wobei Erwärmung zu beobachten ist; $5 g$ Carbazolkalium gaben nach 24 Stunden $4^{1} / 2 g$ Allylcarbazol, das im überschüssigen Allyljodid gelöst war und daraus nach mehrmaligem Umkrystallisieren aus Alkohol in farblosen Rhomben vom Schmelzpunkt $56^{\circ}$ gewonnen wurde. Das Pikrat krystallisiert in schönen roten Nadein vom Schmelzpunkt $86^{\circ}$. (). 2035 g der ïber Schwefelsäure getrockneten Substanz gaben $13 \mathrm{~cm}^{3}$ Stickstofi bei $23^{\circ}$ und $r+t m m$ Druck.

In 100 Teilen:

Berechnet für

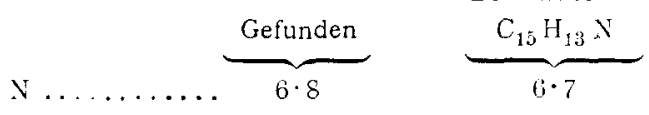




\section{Benzylcarbazol.}

Benzylchlorid wirkt ebenfalls bei Zimmertemperatur heftig auf Carbazolkalium ein; nach 24 stündigem Stehen konnten $5 \mathrm{~g}$ an rohem Benzylcarbazol gewonnen werden. Nach Umkrystallisieren aus Alkohol erhielt ich es in farblosen Nadeln vom Schmelzpunkte $114^{\circ}$. Das Pikrat krystallisiert in schönen roten Nadeln vom Schmelzpunkt $105^{\circ}$.

$0.1776 \mathrm{~g}$ der über Schwefelsäure getrockneten Substanz gaben $9.8 \mathrm{~cm}^{3}$ Stickstoff bei $24^{\circ}$ and $735 \mathrm{~mm}$ Druck.

In 100 Teilen:

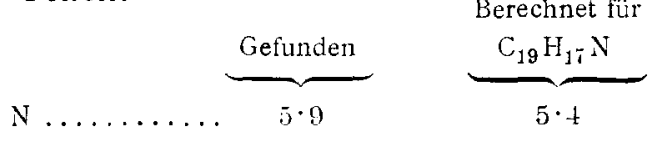

\section{Triphenylmethylcarbazol.}

$7 \mathrm{~g}$ Triphenylmethylchlorid wurden in $50 \mathrm{~cm}^{3}$ trockenem Benzol gelöst, mit $5 g$ Carbazolkalium versetzt und unter zeitweisem Umrühren bis zum gelinden Sieden erwärmt. Nach Erkalten der Lösung wurde vom Niederschlag, der aus Jodkalium und Carbazolkalium bestand, abfiltriert. Auf Zusatz von Petroläther zu der benzolischen Lösung schieden sich ansehnliche Massen eines schwach gelb gefärbten Körpers ab, der nach wiederholtem Umkrystallisieren aus Toluol in farblosen zugespitzten Rhomben vom Schmelzpunkte $245^{\circ}$ erhalten wurde; in Wasser und Alkohol ist er nicht löslich. Die Analyse gab für Triphenylmethylcarbazol gut stimmende Werte.

I. $0 \cdot 1760 g$ der bei $100^{\circ}$ getrockneten Substanz gaben $0 \cdot 6220 g$ Kohlensäure und $0.0921 \mathrm{~g}$ Wasser.

II. $0 \cdot 3040 \mathrm{~g}$ der bei $100^{\circ}$ getrockncten Substanz gaben $9 \cdot 1 \mathrm{~cm}^{3}$ Stickstoff bei $21^{\circ}$ und $734 m m$ Druck.

In 100 Teilen:

\begin{tabular}{|c|c|c|}
\hline 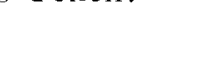 & Gefunden & $\begin{array}{l}\text { Berechnet fur } \\
\mathrm{C}_{31} \mathrm{H}_{23} \mathrm{~N}\end{array}$ \\
\hline$c \ldots \ldots \ldots \ldots$ & $90 \cdot 63$ & $90 \cdot 95$ \\
\hline$H \ldots \ldots \ldots \ldots$ & $5 \cdot 79$ & $5 \cdot 62$ \\
\hline $\mathrm{N} \ldots \ldots \ldots \ldots$ & $3 \cdot 28$ & $3 \cdot 42$ \\
\hline
\end{tabular}


Am Ende der Arbeit sei es mir gestattet, den Herren Professoren Goldschmiedt und Kirpal für das Interesse und die Hilfe, mit der sie mich bei der Durchführung der Arbeit unterstützten, meinen wärmsten Dank auszusprechen. 